\title{
Transient early diastolic murmur and end stage renal disease
}

Sir,

A transient early diastolic murmur may be heard in patients with end stage renal disease. This was initially attributed to aortic regurgitation secondary to fluid overload. ${ }^{1}$ This view was supported by Alexander and Polak, ${ }^{2}$ who described the appearance of the murmur in seven patients with end stage renal failure during episodes of fluid overload; their description of the murmur supports their conclusions that it was of aortic origin.

We have, however, had a patient in end stage renal failure in whom an early diastolic murmur was associated with episodes of typical pericardial pain while he was salt and water depleted. The murmur was loudest when he lay supine and it was clearly audible all over the praecordium. We believe that this observation supports the hypothesis of Barrett et al. ${ }^{3}$ that an early diastolic "murmur" may be a pericardial noise indicative of uraemic pericarditis. This possibility was not mentioned by Alexander and Polak.

Barrett described eight patients and commented that the murmur was not typical of aortic regurgitation in that it was maximal in intensity at and localised to the cardiac apex and became softer when the patient leaned forward. Aortography in six of the patients identified only one patient with possible aortic regurgitation, while echocardiography showed that in all five patients examined a posterior pericardial effusion was present. They concluded that "the early diastolic murmur of renal failure does not usually arise from functional aortic incompetence but may be a sound of pericardial origin". Barrett's conclusions are not supported by Storstein and Örjavik. ${ }^{4}$ They found that in a group of 21 patients with advanced renal failure in whom a diastolic decrescendo murmur was heard, eight had evidence of organic aortic regurgitation and six of the remaining $13 \mathrm{had}$ aortograms which showed that four had aortic regurgitation which was probably functional.

The origin of the early diastolic murmur associated with renal failure is therefore still in doubt. It is possible that it is related to functional aortic regurgitation in some patients and to uraemic pericarditis in others. It is important to identify cases of pericarditis at an early stage since it is an indication for urgent dialysis and a contraindication to anticoagulation because of the dangers of cardiac tamponade. It is possible that a pericardial friction rub may be mistaken for an aortic early diastolic murmur. When an early diastolic murmur is observed in chronic renal failure, the possibility that it is pericardial in origin should be considered, especially if it is not typical of aortic regurgitation.

Malcolm VandenBurg, Rodney Foale,

The London Hospital, (Whitechapel).

\section{References}

1 Adam WR, Dawborn JK, Rosenbaum M. Transient early diastolic murmurs in patients with renal failure. Med f Aust 1970; 2: 1085-6.

2 Alexander WD, Polak A. Early diastolic murmurs in end-stage renal failure. $B r$ Heart $\mathcal{f}$ 1977; 39: 900-2.

3 Barrett LJ, Robinson MA, Whitford JA, Lawrence JR. The diastolic murmur of renal failure. $N$ Engl f Med 1976; 295: 121-4.

4 Storstein O, Örjavik O. Aortic insufficiency in chronic renal failure. Acta Med Scand 1978; 203: 175-80.

This letter was shown to $\mathrm{Dr}$ Alexander and Professor Polak who comment as follows.

Sir,

We agree that there may be occasions when a pericardial friction rub mimics an aortic diastolic murmur, and this should be suspected particularly if a patient with renal failure is salt and water depleted (our patients with functional aortic regurgitation were all overloaded with salt and water). The dilemma about heparinising the patient, however, is unlikely to arise often, and we believe that these patients can safely be heparinised if there is no clinical evidence of pericarditis other than a murmur confined to early diastole and heard best at the aortic area. Echocardiography may disclose the presence of pericardial fluid in patients with advanced renal failure, but will not resolve the dilemma. ${ }^{1}$ We feel sure that Drs VandenBurg and Foale would agree that aortography should be reserved for the rare patient with renal failure whose murmur persists after overhydration has been corrected.

W D Alexander,

A Polak,

Saint Mary's Hospital, Portsmouth.

\section{Reference}

1 Goldstein DH, Nagar C, Srivastava N, Schacht RA, Ferris FZ, Flowers NC. Clinically silent pericardial effusions in patients on long-term haemodialysis. Chest 1977; 72: 744-7. 